\title{
Use of Turbine-level Data for Improved Wind Power Forecasting
}

\author{
Jethro Browell, Member, IEEE, Ciaran Gilbert, Student Member, IEEE and David McMillan, Member, IEEE \\ University of Strathclyde, Glasgow, UK \\ \{jethro.browell, ciaran.gilbert, d.mcmillan\}@strath.ac.uk
}

(C)2017 IEEE. Personal use of this material is permitted. Permission from IEEE must be obtained for all other uses, in any current or future media, including reprinting/republishing this material for advertising or promotional purposes, creating new collective works, for resale or redistribution to servers or lists, or reuse of any copyrighted component of this work in other works. This work has been accepted for publication in the proceedings of the IEEE PowerTech Conference, Manchester, UK, 2017.

Abstract-Short-term wind power forecasting is based on modelling the complex relationship between the weather forecasts and wind farm power production. To date, efforts to improve wind power forecasts have focused on improving Numerical Weather Prediction and wind farm power curve models. However, utilityscale wind farms cover large areas meaning that a single power curve model may struggle to represent the collective behaviour of large numbers of wind turbines. Contemporary statistical techniques are capable of processing large volumes of data, enabling the assimilation of measurements from individual wind turbines to construct a more detailed representation of wind farm power generation. Here, three state-of-the-art techniques are applied to forecast wind farm power production 1) directly from numerical weather predictions, and 2) by aggregating forecasts for individual wind turbines. Furthermore, it is observed that some wind turbines are better predictors than others and an aggregation process based on conditional weighting is proposed. In case studies of two large wind farms in the UK, it is shown that wind farm power forecasts comprising a conditional weighted sum of turbine-level predictions are superior to a direct wind farm forecast for horizons up to $\mathbf{4 8}$ hours ahead. Specifically, performance of the best-performing benchmark, the gradient boosting machine, is improved by $12 \%$ at Clyde South wind farm and by $6 \%$ at Gordonbush.

Index Terms-Wind Power Forecasting, Big Data, Machine Learning, LASSO, Gradient Boosting

\section{INTRODUCTION}

Wind power forecasting is an integral component of modern power system operation and electricity market participation in areas with a significant penetration of wind generation. The stochastic nature of the wind resource means that forecasts are required to inform decisions where future generation is a factor. Forecast from days to a week ahead are valuable when scheduling conventional generation and maintenance on wind farms, day-ahead forecasts are required for participants in electricity markets, and intra-day forecasts are used by participants in short-term markets and to power system operators who must balance supply and demand in real time [1], [2].
Deterministic wind power forecasting, comprising singlevalued estimates of future power, is approaching technological maturity following a concerted research effort reviewed comprehensively in [3], [4]. At present there are many commercial providers offering deterministic wind power forecasts. However, there is a broad consensus in the academic community that wind power should be modelled as a stochastic process and that forecasts should be probabilistic in order to quantify forecast uncertainty [5], [6]. That said, many forecast users still only utilise single-valued forecasts due to the difficulty of incorporating complex probabilistic information into decisionmaking processes in practice.

There are two sources of error in wind power forecasting: meteorological forecast errors, and errors introduced by the wind-to-power conversion process. In this work we attempt to reduce the later. Typically, wind-to-power conversion is modelled at the farm level; these models may contain tens or hundreds of input features derived from meteorological forecasts and lagged measurements. Approaches based on boosted regression trees [7], [8], linear regression with sparsity [9] and neural networks [10] are at the forefront of the technology, with gradient boosting methods winning both the 2012 and 2014 Global Energy Forecasting Competitions [11], [12]. However, large wind farms contain many turbines distributed over a wide geographical area with each turbine experiencing different conditions. Here we investigate the utilisation of turbine-level power production an propose a bottom-up hierarchical forecasting methodology, an idea which has proved successful in other applications [13]-[15].

The power generated by wind turbines is routinely measured and transmitted to operators making this data available for use in forecasting systems. Here, we test the hypothesis that power forecasts for large wind farms can be improved by modelling and combining the wind-to-power conversion process for individual spatially distributed turbines. We will draw upon developments in large-scale spatio-temporal forecasting such as [16], [17] and investigate the ability of modern machine learning algorithms to efficiently model these processes and aggregate the resulting forecasts.

\section{Forecasting Methodology}

Two model structures are considered: first, wind farm power production modelled directly on numerical weather predictions (NWP) and lagged power production, and second, a two stage process where individual turbine production is forecast based 
on NWP and lagged power measurements and then aggregated to form a prediction of the total wind farm production. Forecasts are produced from 30 minutes to 48 hours ahead in 30 minute intervals.

Three state-of-the-art forecasting techniques are implemented to forecast wind farm and wind turbine power production. First is linear regression with sparse parameter estimation using the least absolute shrinkage and selection operator (LASSO) [18]. LASSO simultaneously performs parameter estimation and feature selection enabling the user to engineer many features and then rely on the estimator to deselect some features with parameters estimates equalling exactly zero. Second is the gradient boosting machine (GBM) [19] which is a tree-based method for non-linear function approximation, and third is the extreme gradient boosting machine, which is an extension of the GBM [20]. The LASSO and GBM have pedigree as the basis of winning entries in the wind power forecasting track of the Global Energy Forecasting Competition 2014, and the latter in particular for winning many other machine learning competitions [12].

\section{A. Least Squares LASSO}

A linear model for the quantity $y_{t}$ we are attempting to forecast is the weighted sum of input features $\mathbf{x}_{t}=\left[x_{1, t}, \ldots, x_{p, t}\right]$ plus an error term $\epsilon_{t}$,

$$
y_{t}=\sum_{i=1}^{p} \beta_{i} x_{i, t}+\epsilon_{t}
$$

where $\boldsymbol{\beta}=\left[\beta_{1}, \ldots, \beta_{p}\right]^{\mathrm{T}}$ are unknown parameters to be estimated.

For a set of $T$ samples, $\{\mathbf{Y}, \mathbf{X}\}$, where $\mathbf{Y}$ and $\mathbf{X}$ are matrices of vertically stacked instances of $y_{t}$ and $\mathbf{x}_{t}$, respectively, the least squares estimate of $\boldsymbol{\beta}$ is the solution to the problem

$$
\underset{\boldsymbol{\beta}}{\operatorname{argmin}}\left\{\|\mathbf{Y}-\mathbf{X} \boldsymbol{\beta}\|_{2}^{2}\right\} \text {. }
$$

Large numbers of features, and possible multicollinearity, and can lead to poor parameter estimates and predictive performance, as well as making model interpretation difficult [18]. There is therefore a need to regularise the estimation process. LASSO achieves this by penalising the $\ell_{1}$ norm of $\boldsymbol{\beta}$. The lasso estimation problem is given by

$$
\underset{\boldsymbol{\beta}}{\operatorname{argmin}}\left\{\frac{1}{2 T}\|\mathbf{Y}-\mathbf{X} \boldsymbol{\beta}\|_{2}^{2}+\lambda\|\boldsymbol{\beta}\|_{1}\right\} \quad .
$$

The user-defined shrinkage parameter $\lambda$ controls sparsity and is typically selected via a cross-validation procedure.

Due to the non-linear nature of the relationship between wind speed and power, and others, it is necessary to construct features which capture these effects. This can be achieved by recasting (1) as an additive model

$$
y_{t}=\sum_{i=1}^{p} \beta_{i} f_{i}\left(x_{i, t}\right)+\epsilon_{t},
$$

where $f_{i}(\cdot)$ are smooth functions chosen to capture non-linear effects such as the familiar power curve. The capability of
LASSO to perform feature selection makes this convenient as multiple functions $f_{i}(\cdot)$ can be included but only those which add the most value are retained in the final model.

\section{B. Tree-based Gradient Boosting Machine}

A regression tree with $J$ leaves and weights $w_{j}$ has the additive form

$$
k\left(\mathbf{x} ; \mathcal{T}=\left\{w_{j}, R_{j}\right\}^{J}\right)=\sum_{j=1}^{J} w_{j} I\left(\mathbf{x} \in R_{j}\right)
$$

where $R_{j}$ are disjoint regions that collectively cover the input space spanned by $\mathbf{x}$, and $I(\cdot)$ is the indicator function. Individual trees can be fit very efficiently using the a process of recursive partitioning but have limited predictive power and for this reason are often called weak learners [21]. Gradient boosting attempts to overcomes this drawback by constructing a 'stronger' learner from an ensemble of weak learners.

The gradient boosting machine $F_{n}\left(\mathbf{x}_{t}\right)$ is the sum of $n$ weak learners

$$
\begin{aligned}
y_{t} & =F_{n}\left(\mathbf{x}_{t}\right)+\epsilon_{t} \\
& =\sum_{i=1}^{n} f_{i}\left(\mathbf{x}_{t}\right)+\epsilon_{t}
\end{aligned}
$$

where, in this case, each $f_{i}\left(\mathbf{x}_{t}\right)$ is a regression tree. The ensemble of regression trees is constructed sequentially by estimating the new regression tree $f_{n+1}\left(\mathbf{x}_{t}\right)$ via

$$
\underset{f_{n+1}}{\operatorname{argmin}} \sum_{t} L\left(y_{t}, F_{n}\left(\mathbf{x}_{t}\right)+f_{n+1}\left(\mathbf{x}_{t}\right)\right)
$$

for some loss function $L(\cdot)$. Where $L(\cdot)$ is differentiable, this optimisation can be solved by steepest descent written

$$
\begin{aligned}
g_{n}\left(\mathbf{x}_{t}\right) & =\frac{\partial L\left(y_{t}, F_{n}\left(\mathbf{x}_{t}\right)\right)}{\partial F_{n}} \\
f_{n+1}\left(\mathbf{x}_{t}\right) & =-\rho_{n} g_{n}\left(\mathbf{x}_{t}\right)
\end{aligned}
$$

where

$$
\rho_{n}=\underset{\rho}{\operatorname{argmin}} \sum_{t} L\left(y_{t}, F_{n}\left(\mathbf{x}_{t}\right)-\rho g_{n}\left(\mathbf{x}_{t}\right)\right) \quad .
$$

For a finite dataset, a regression tree $k(\mathbf{x} ; \mathcal{T})$ is fit to the psudoresiduals $g_{n}\left(\mathbf{x}_{t}\right)$ by solving

$$
\underset{\mathcal{T}, \gamma}{\operatorname{argmin}} \sum_{t}\left[-g_{n}\left(\mathbf{x}_{t}\right)+\gamma k\left(\mathbf{x}_{t} ; \mathcal{T}\right)\right]^{2} .
$$

The process of recursive partitioning makes estimating each new regression tree computationally inexpensive. The user must specify the number of trees to fit, $n$, and the number of regions each tree divides the input space into. An additional shrinkage parameter may be included in (10) to control the learning rate of the fitting procedure and reduce the impact if individual trees. 


\section{Extreme Gradient Boosting Machines}

A recent advance gradient boosting has seen the development of Extreme Gradient Boosting (XGBoost) algorithm, which solves the optimisation problem (8) by performing second order gradient descent [20]. This approach results in more efficient model fitting making it more scalable than traditional gradient boosting, and is included here for comparison. For details of the fitting algorithm see [20].

\section{Wind POWER ForeCASTING}

As a benchmark, the methodologies described above are implemented to produce power forecasts for wind farms directly, the conventional approach to wind power forecasting, where $y_{t}$ is the total power produced by the wind farm at time $t$. Motivated by knowledge that wind conditions can vary significantly across large wind farms and intuition that different turbines will be better predictors than others at different times, we use the same methodologies to forecast production for individual wind turbines which are then combined to produce a farm-level forecast. In both cases $\mathbf{x}_{t}$ contains features from NWP, namely wind speed and direction at $10 \mathrm{~m}, 80 \mathrm{~m}$ and $100 \mathrm{~m}$ above ground linearly interpolated to 30 -minute resolution, and lagged power measurements for the most recent 48 halfhour periods. Bilinear interpolation is used to obtain single values for each meteorological variable at the location of the target wind farm from the closes four NWP grid points.

In order to capture non-linear features of the wind-power relationship in the linear model (4) a series of threshold functions are also included. Specifically

$$
f_{\max }^{(c)}\left(x_{i, t}\right)=\max \left\{c, x_{i, t}\right\} \quad, \quad c=2,4, \ldots, 24 .
$$

These additional features are not used in the tree-methods as they would be redundant. In all models time-of-day features included to capture and diurnal bias in the NWP and are modelled by cubic spline kernels, along similar lines to [22].

\section{A. Aggregating Turbine-level Forecasts}

Two approaches to forecast aggregation are considered for the novel turbine level methodology. In the first, the forecast for the wind farm as a whole is given by the weighted sum of individual turbine forecasts. The second is also based on a weighted sum of individual turbine forecasts but in this case the weights are conditioned on the forecast wind direction. This approach was driven by the idea that different combinations of turbines will be better predictors of total wind farm generation depending on the incident wind direction due to the array layout and local topographic effects. For the simple weighted sum (WS)

$$
y_{t}=\sum_{i=1}^{N} \beta_{i} x_{i, t}+\epsilon_{t}
$$

where here $x_{i, t}$ is the forecasts power a the $i^{\text {th }}$ turbine at time $t$ and the parameters $\beta_{i}$ are estimated by the LASSO (3).

In order to condition the weighting on wind direction, separate parameters are estimated for different wind directions

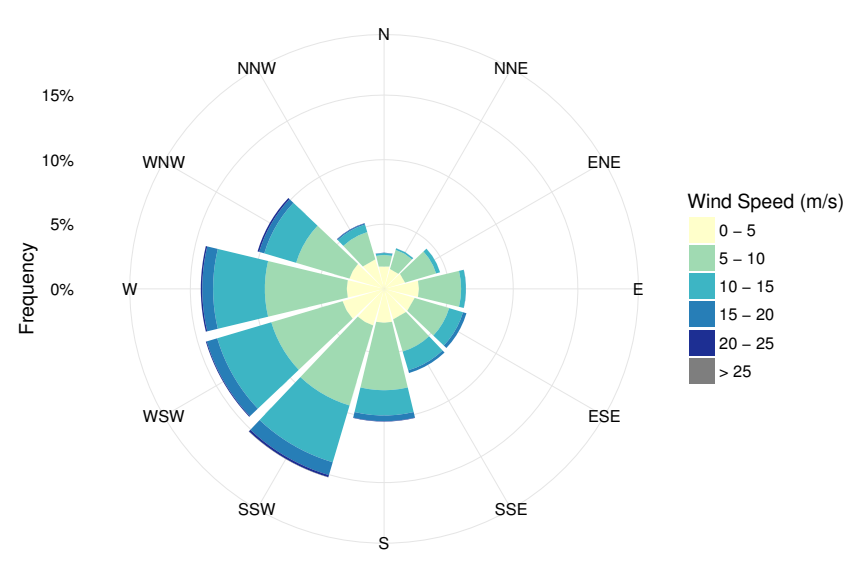

Fig. 1. Wind rose for forecast wind speed and meteorological wind direction at $100 \mathrm{~m}$ above ground for Clyde South from training dataset.

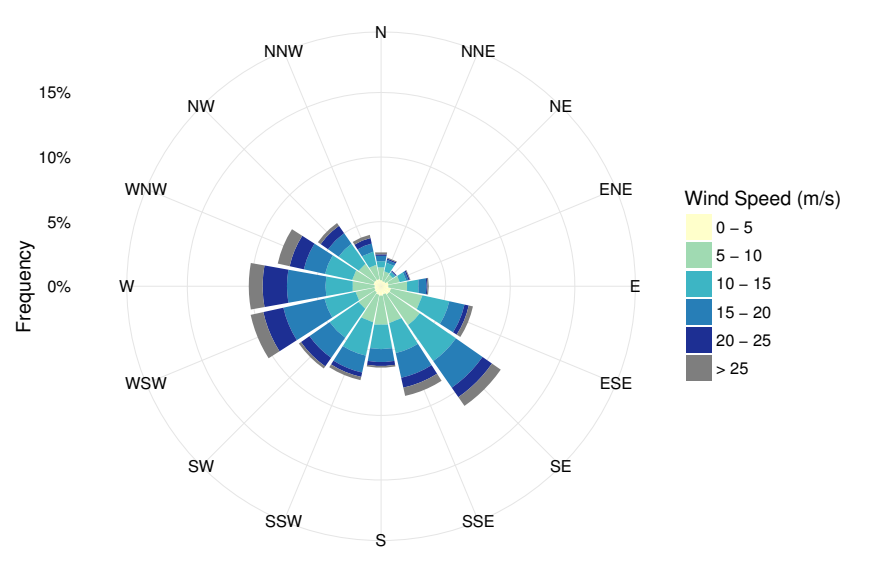

Fig. 2. Wind rose for forecast wind speed and meteorological wind direction at $100 \mathrm{~m}$ above ground for Gordonbush from training dataset.

and the weighted sum (14) becomes the conditional weighted sum (CWS)

$$
y_{t}=\sum_{i=1}^{N} \omega_{i}(\theta) x_{i, t}+\epsilon_{t}
$$

For practical reasons, data are grouped into a fixed number of bins (determined by $k$-fold cross-validation) and $\omega_{i}(\theta)$ are estimated for each bin. Parameters are then estimated by

$$
\boldsymbol{\omega}(\theta)=\underset{\boldsymbol{\beta}}{\operatorname{argmin}}\left\{\frac{1}{2 T}\left\|\mathbf{Y}_{\theta}-\mathbf{X}_{\theta} \boldsymbol{\beta}\right\|_{2}^{2}+\lambda\|\boldsymbol{\beta}\|_{1}\right\}
$$

where $\mathbf{Y}_{\theta}$ and $\mathbf{X}_{\theta}$ are matrices of vertically stacked instances of $y_{t}$ and $\mathbf{x}_{t}$ where the forecast wind direction at time $t$ lies in the same bin as $\theta$.

\section{CASe Study}

The benchmark and proposed methods are tested on two large UK wind farms operated by SSE. Details are listed in Table I. Generation data from individual turbines' SCADA 
system and the wind farm power export meter are used at 30 minute resolution with instances of curtailment flagged and excluded from the forecasting exercise. Numerical Weather Forecasts from the Global Forecasting System [23] are used and comprise wind speed and direction at $80 \mathrm{~m}$ and $100 \mathrm{~m}$ above ground. The methodologies described are implemented in R using the packages glmnet, gbm, and xgboost [20], [24]-[26].

\section{REsUlts}

The LASSO, GBM, and XGBoost predictors and set-up to 1) forecast wind farm power production directly, and 2) forecast the power production of individual turbines. In the second case, the forecast for the wind farm as a whole is produced by a simple weighted sum (WS) and alternatively a conditional weighted sum (CWS) of turbine forecasts, with weights estimated using least squares LASSO.

To evaluate forecasts performance two metrics were used: the mean absolute error (MAE) and root mean squared error (RMSE). The RMSE across all forecast horizons was used to inform model selection and performance is evaluated by horizon in order to illustrate how forecast performance degrades with lead-time. These metrics are given by

$$
\begin{aligned}
\text { MAE } & =\frac{1}{N} \sum_{i=1}^{N}\left|y_{i}-\hat{y}_{i}\right| \\
\text { RMSE } & =\sqrt{\frac{1}{N} \sum_{i=1}^{N}\left(y_{i}-\hat{y}_{i}\right)^{2}}
\end{aligned}
$$

where $\hat{y}_{i}$ is the forecast of $y_{i}$.

The parameters governing the fitting process of each forecast method and the number of direction bins were determined by $k$-fold cross validation with five folds on the training data for each wind farm. The resulting direction bins are illustrated in Figure 1 and Figure 2 for each wind farm.

The computational time for 5-fold cross-validation at Gordonbush was 6, 880, and 300 seconds for the LASSO, GBM, and XGBoost, respectively. This illustrates the important tradeoff between model accuracy and computational time between each of the methodologies. Additionally, the GBM and extreme gradient boosting machine methods may controlled by several parameters (including learning rate, interaction depth and number of trees) whereas the only parameter for crossvalidation in the LASSO is the shrinkage.

Performance of the LASSO, GBM, and XGBoost methods are plotted in Figure 3 for Clyde South. The performance of all three methods is improved across all horizons when utilising turbine-level information with the conditional weighted-sum method. At this wind farm both variants of boosted machines perform similarly with the turbine level data included up to a horizon of $24 \mathrm{~h}$, with the GBM method performing best overall when performance is averaged across all forecast horizons. Full results are listed in Table II. The most improved method with the incorporation of the turbine level data is the LASSO model which shows a $17 \%$ reduction in RMSE when averaged

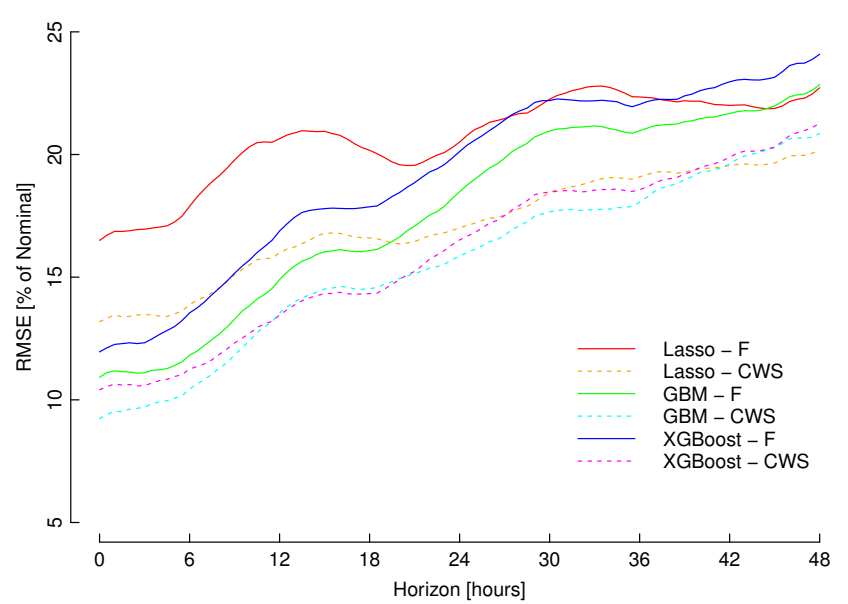

Fig. 3. Forecast performance at Clyde South based on wind farm level data only $(-\mathrm{F})$ and wind turbine level data with a conditional weighted sum (CWS)

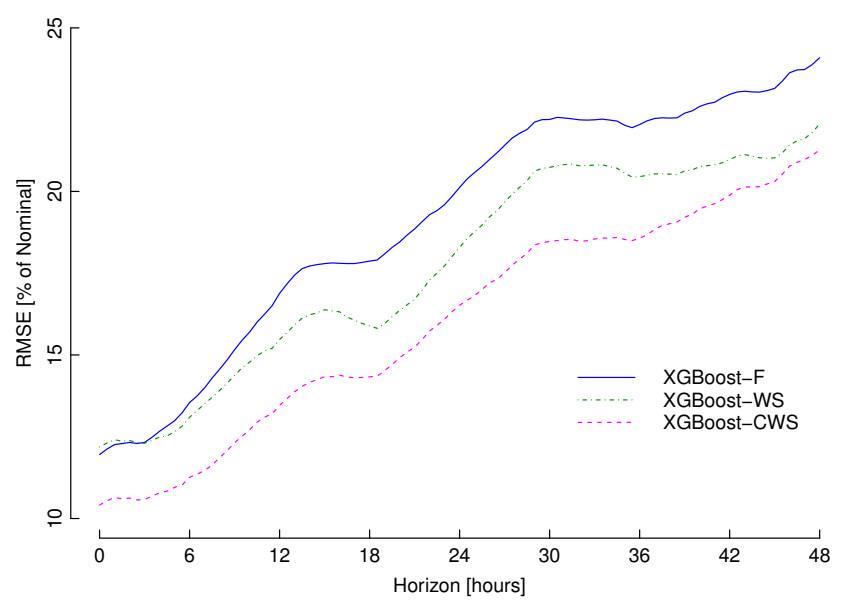

Fig. 4. Forecast performance at Clyde South for the XGBoost method based on wind farm level data only $(-\mathrm{F})$, turbine level data with a weighted sum (-WS), and wind turbine level data with a conditional weighted sum (-CWS).

across all horizons. Results for all methods are tabulated in Table III.

The value of conditioning the aggregation of individual turbine forecasts on wind direction is illustrated in Figure 4 which examines the XGBoost methodology at Clyde South. Here the simple weighted sum performs well compared to the wind farm level data and gives an appreciable improvement across forecast horizons beyond 3 hours. However, the greater value of the turbine level approach is realised through the conditional weighted sum method which demonstrates consistent improvement across all horizons compared to the wind farm level data only. Averaged across all forecast horizons it leads to a $16 \%$ reduction in the RMSE and $12 \%$ in MAE compared to the standard wind farm level approach.

Forecast performance for Gordonbush is plotted in Figure 5. It can be seen that with a more complex wind rose, shown in Figure 2, the forecast quality is lower than that of Clyde 
TABLE I

DETAILS OF WIND FARMS USED IN CASE STUDY. TRAINING AND TEST PERIODS DIFFER DUE TO DIFFERENCES IN DATA AVAILABILITY ONLY.

\begin{tabular}{l||cccccc}
\hline Wind Farm & Terrain & Area & Number of Turbines & Turbine Rating & Training Period & Test Period \\
\hline Clyde South & Complex & $20 \mathrm{~km}^{2}$ & 56 & $2.3 \mathrm{MW}$ & 22 months & 6 months \\
Gordonbush & Complex & $15 \mathrm{~km}^{2}$ & 35 & $2 \mathrm{MW}$ & 17 months & 6 months \\
\hline
\end{tabular}

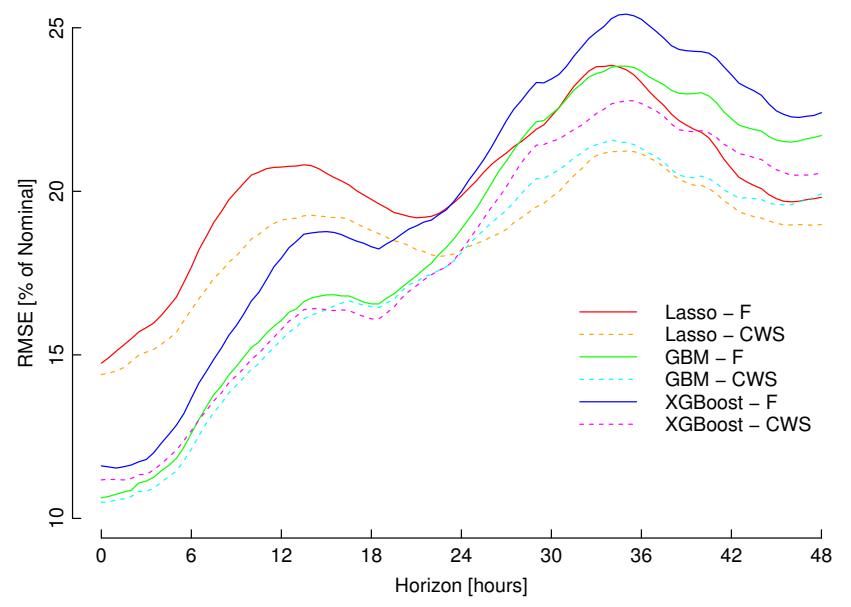

Fig. 5. Forecast performance at Gordonbush based on wind farm level data only $(-\mathrm{F})$ and wind turbine level data with a conditional weighted sum (CWS).

South, possibly as a result of local effects which are not captured by the relatively low resolution NWP. However, the performance of all methods are improved when utilising turbine-level information, although to a lesser extent. At Clyde South the RMSE reduction was between $12 \%$ and $17 \%$ where as at Gordonbush it is between $6 \%$ and $9 \%$. For both wind farms GBM with CWS had the lowest MAE and RMSE overall.

A summary of the results across both wind farms is shown in Table II where the error metrics have been averaged across all forecast horizons. The simple weighted sum of forecast turbine power generation led to consistent improvements in the averaged RMSE at both wind farms, with the XGBoost method showing the most improvement. However, the weighted sum of turbine forecasts conditional on wind direction improved overall performance to a greater extent across all methods and wind farms.

\section{Conclusions}

The use of turbine level data for improved wind forecasting has been studied and evaluated using a case study of two wind farms with differing characteristics. Three state-of-theart techniques - linear regression (additive model) estimated via LASSO, gradient boosting machines, and extreme gradient boosting machines - were applied to forecast wind farm power production directly from numerical weather predictions, and by aggregating forecasts made in the same way but for individual wind turbines.

On a case study comprising two large wind farms considering forecast up to 48 hours ahead, it is shown that aggregating
TABLE II

SUMMARY RESULTS FOR DIRECT WIND FARM LEVEL FORECASTS (F), TURBINE LEVEL WITH WEIGHTED SUM (WS), AND TURBINE LEVEL WITH CONDITIONAL WEIGHTED SUM (CWS). THE BEST PERFORMING AGGREGATION METHOD FOR EACH FORECASTING APPROACH IS ITALICISED, AND BEST OVERALL PERFORMANCES ARE EMBOLDENED.

\begin{tabular}{|c|c|c|c|c|c|}
\hline \multirow{2}{*}{ Model } & \multirow{2}{*}{ Aggregation } & \multicolumn{2}{|c|}{ Clyde South } & \multicolumn{2}{|c|}{ Gordonbush } \\
\hline & & RMSE & MAE & RMSE & MAE \\
\hline \multirow{3}{*}{ LASSO } & $\mathrm{F}$ & 20.69 & 14.33 & 20.20 & 15.21 \\
\hline & WS & 20.63 & 14.62 & 20.16 & 15.59 \\
\hline & CWS & 17.16 & 12.76 & 18.68 & 14.76 \\
\hline \multirow{3}{*}{ GBM } & F & 17.74 & 12.70 & 18.57 & 13.76 \\
\hline & WS & 17.61 & 12.44 & 18.53 & 13.98 \\
\hline & CWS & 15.62 & 11.19 & 17.40 & 13.25 \\
\hline \multirow{3}{*}{ XGBoost } & $\mathrm{F}$ & 19.19 & 13.55 & 19.88 & 14.63 \\
\hline & WS & 17.77 & 12.97 & 18.93 & 14.60 \\
\hline & CWS & 16.06 & 11.96 & 18.02 & 14.05 \\
\hline
\end{tabular}

TABLE III

PERCENTAGE REDUCTION IN RMSE COMPARED TO DIRECT WIND FARM FORECASTS FOR AGGREGATED TURBINE LEVEL FORECASTS WITH WEIGHTED SUM (WS), AND CONDITIONAL WEIGHTED SUM (CWS)

\begin{tabular}{lc||c|c}
\hline Model & Aggregation & Clyde South & Gordonbush \\
\hline \multirow{2}{*}{ LASSO } & WS & 0.29 & 0.20 \\
& CSW & 17.05 & 7.52 \\
GBM & WS & 0.68 & 0.20 \\
& CWS & 11.94 & 6.30 \\
\multirow{2}{*}{ XGBoost } & WS & 7.37 & 4.74 \\
& CWS & 16.33 & 9.36 \\
\hline
\end{tabular}

turbine-level forecasts gives an improved overall wind farm production forecast. Furthermore, the aggregation process is improved if conditioned on wind direction. It was found that gradient boosting machine produced forecasts with the lowest RMSE and MAE, reducing RMSE over direct wind farm-level forecasting by $12 \%$ for the Clyde South wind farm, and $6 \%$ for Gordonbush, which has a more complex wind rose.

Future work should consider the utility of this approach in combination with downscaled NWP and if similar improvements are possible on very-short time scales where statistical methods typically out perform those based on NWP. Another consideration should be the use of turbine-level forecasting in a probabilistic setting.

\section{ACKNOWLEDGEMENTS}

The authors thanks SSE plc for the supply of wind farm data and NOAA/GFS for the supply of meteorological data. Jethro Browell is supported by the University of Strathclyde's 
EPSRC Doctoral Prize, grant number EP/M508159/1, and Ciaran Gilbert by the University of Strathclyde's EPSRC Centre for Doctoral Training in Wind and Marine Energy Systems, grant number EP/L016680/1.

Data Statement: Due to confidentiality agreements with research collaborators, access to wind power data is restricted. Numerical weather predictions are from GFS and are available at www.ncdc.noaa.gov.

\section{REFERENCES}

[1] T. Ackermann, Ed., Wind power in power systems, 2nd ed. John Wiley \& Sons: New York, 2012

[2] J. Morales, A. Conejo, H. Madsen, P. Pinson, and M. Zugno, Integrating Renewable in Electricity Markets. Springer, 2014.

[3] C. Monteiro, R. Bessa, V. Miranda, A. Botterud, J. Wang, and G. Conzelmann, "Wind power forecasting: State-of-the-art 2009," Argonne National Laboratory ANL/DIS-10-1, Tech. Rep., 2009.

[4] G. Giebel, R. Brownsword, G. Kariniotakis, M. Denhard, and C. Draxl, The State-of-the-Art in Short-Term Prediction of Wind Power. ANEMOS.plus, 2011, project funded by the European Commission under the 6th Framework Program, Priority 6.1: Sustainable Energy Systems.

[5] P. Pinson, "Wind energy: Forecasting challenges for its operational management," Statistical Science, vol. 28, no. 4, pp. 564-585, 2013.

[6] Y. Zhang, J. Wang, and X. Wang, "Review on probabilistic forecasting of wind power generation," Renewable and Sustainable Energy Reviews, vol. 32, no. 0, pp. 255-270, 2014. [Online]. Available: http://www.sciencedirect.com/science/article/pii/S1364032114000446

[7] L. Silva, "A feature engineering approach to wind power forecasting: \{GEFCom\} 2012," International Journal of Forecasting, vol. 30, no. 2 , pp. 395-401, 2014.

[8] M. Landry, T. P. Erlinger, D. Patschke, and C. Varrichio, "Probabilistic gradient boosting machines for GEFCom2014 wind forecasting," International Journal of Forecasting, vol. 32, no. 3, pp. 1061-1066, 2016.

[9] F. Ziel, C. Croonenbroeck, and D. Ambach, "Forecasting wind power modeling periodic and non-linear effects under conditional heteroscedasticity," Applied Energy, vol. 177, pp. 285-297, 2016.

[10] D. Lee and R. Baldick, "Short-term wind power ensemble prediction based on gaussian processes and neural networks," Smart Grid, IEEE Transactions on, vol. 5, no. 1, pp. 501-510, Jan 2014.

[11] T. Hong, P. Pinson, and S. Fan, "Global energy forecasting competition 2012," International Journal of Forecasting, vol. 30, pp. 357-363, 2014.
[12] T. Hong, P. Pinson, S. Fan, H. Zareipour, A. Troccoli, and R. J. Hyndman, "Probabilistic energy forecasting: Global energy forecasting competition 2014 and beyond," International Journal of Forecasting, 2016, in press.

[13] B. J. Dangerfield and J. S. Morris, "Top-down or bottom-up: Aggregate versus disaggregate extrapolations," International Journal of Forecasting, vol. 8, pp. 233-241, 1992.

[14] R. J. Hyndman, R. A. Ahmed, G. Athanasopoulos, and H. L. Shang, "Optimal combination forecasts for hierarchical time series," Computational Statistics \& Data Analysis, vol. 55, no. 9, pp. 2579-2589, 2011.

[15] T. Hong, P. Wang, and L. White, "Weather station selection for electric load forecasting," International Journal of Forecasting, vol. 31, no. 2, pp. 286-295, 2015.

[16] J. Dowell and P. Pinson, "Very-short-term probabilistic wind power forecasts by sparse vector autoregression," IEEE Transactions on Smart Grid, vol. 7, no. 2, pp. 763-770, March 2016.

[17] R. Bessa, A. Trindade, A. Monteiro, V. Miranda, and C. S. P. Silva, "Solar power forecasting in smart grid using distributed information," in 18th Power Systems Computation Conference, 2014.

[18] T. Hastie, R. Tibshirani, and M. Wainwright, Statistical Learning with Sparsity: the Lasso and Generalizations, ser. Monographs on Statistics \& Applied Probability. Chapman and Hall/CRC, 2015.

[19] J. Friedman, "Greedy function approximation: a gradient boosting machine," Annals of Statistics, vol. 29, no. 5, pp. 1189-1232, 2001.

[20] T. Chen and C. Guestrin, "XGBoost: A Scalable Tree Boosting System," ArXiv e-prints, June 2016.

[21] L. Breiman, J. Friedman, C. J. Stone, and R. Olshen, Classification and Regression Trees. Chapman \& Hall/CRC, 1984.

[22] F. Ziel, R. Steinert, and S. Husmann, "Efficient modeling and forecasting of the electricity spot price," Energy Economics, vol. 47, pp. 98-111, 2015.

[23] NOAA National Centers for Environmental Prediction, "NOAA/NCEP Global Forecast System (GFS) Atmospheric Model," June 2011, accessed May 2016.

[24] R Core Team, R: A Language and Environment for Statistical Computing, R Foundation for Statistical Computing, Vienna, Austria, 2016. [Online]. Available: https://www.R-project.org/

[25] J. Friedman, T. Hastie, and R. Tibshirani, "Regularization paths for generalized linear models via coordinate descent," Journal of Statistical Software, vol. 2010, no. 1, pp. 1-22, 33.

[26] G. Ridgeway and with contributions from others, gbm: Generalized Boosted Regression Models, 2015, r package version 2.1.1. [Online].

Available: https://CRAN.R-project.org/package $=\mathrm{gbm}$ 